\title{
La salud sexual y reproductiva en las mujeres de la comunidad de Siape (Barranquilla) y los pronunciamientos de la Corte constitucional de Colombia ${ }^{1}$
}

\author{
Sexual and reproductive health in women of the community Siape \\ (Barranquilla) and the pronouncements of the Constitutional Court of \\ Colombia
}

\author{
Autores: Rosario Joaquín Reales Vega ${ }^{2}$ \\ Juan Carlos Berrocal Durán ${ }^{3}$ \\ Correspondencia: rosario.reales@curnvirtual.edu.co \\ juan.berrocal@curnvirtual.edu.co
}

\begin{abstract}
RESUMEN
El objetivo central de este artículo de investigación se constituyó en caracterizar la salud sexual y reproductiva de las mujeres del barrio Siape de la ciudad de Barranquilla, con el propósito de implementar estrategias que puedan contribuir en mejorar la calidad de vida. Como Objetivos Específicos se propusieron: determinar la mortalidad materna del barrio Siape y sus causas para implementar acciones socioculturales y socioeducativas que contribuyan a disminuir la situación; establecer el tipo de controles que realizan las mujeres embarazadas y el tratamiento que se les indica en estos; identificar los diferentes métodos de planificación familiar utilizados por la población de mujeres en el sector para la prevención de embarazos no deseados. El Método utilizado fue el cuantitativo, con la realización de una investigación de tipo descriptivo, utilizando una población constituida por las mujeres residentes en el Barrio Siape de la ciudad de Barranquilla, con edades entre los 13 y los 60 años. La muestra utilizada fue no probabilística voluntaria. Entre las conclusiones de la investigación se tienen los siguientes aspectos: en las comunidades más vulnerables se ve reflejado el poco cuidado de las mujeres en estado de embarazo, y el tenue acompañamiento por parte de los familiares en la realización de los controles y en el cuidado de ellas.
\end{abstract}

\section{Palabras Clave.}

Salud Sexual, Salud Reproductiva, Mujeres, Parto, Mortalidad Materna.

\footnotetext{
${ }^{1}$ Este Articulo es resultados del proyecto de investigación "Caracterización de la salud sexual y reproductiva de las mujeres del Barrio Siape de la ciudad de Barranquilla, adscrito a la Línea de Investigación Sociedad Cultura y Educación desarrollada por los Grupos de Investigación Andrés Bello del Semillero Rafael Wenceslao Núñez Moledo del programa de derecho y el Grupo de investigación Societas de Trabajo social de la Corporación Universitaria Rafael Núñez Sede Barranquilla. Juan Carlos Berrocal Duran y Rosario Joaquín Reales Vega son los investigadores responsables de este proyecto, y cuenta con la participación de los estudiantes Kevin Patrón Ayola, Yoismar Cabarcas Vizcaíno y Janeris Mercado Martínez del Programa de Trabajo Social. El proyecto fue financiado en su totalidad por la Corporación Universitaria Rafael Núñez

${ }^{2}$ Sociólogo. Especialista en Gestión de Proyectos Educativos. Maestreando en Educación de la Universidad Simón Bolívar. Líder del Grupo de Investigaciones Societas del Programa de Trabajo Social de la Corporación Universitaria Rafael Nuñez sede Barranquilla Docente Programa de Trabajo Social y Derecho Barranquilla.

3 Abogado. Especialista en Derecho Laboral. Magíster en Derecho Procesal de la Universidad Simón Bolívar. Doctorante en Ciencias Políticas. Líder del Grupo de Investigaciones Andrés Bello, del Programa de Derecho de la Corporación Universitaria Rafael Nuñez sede Barranquilla Docente Programa de Trabajo Social y Derecho Barranquilla..
} 


\begin{abstract}
The central objective of this research article was to characterize the sexual and reproductive health of the women of the Siape neighborhood of the city of Barranquilla, with the purpose of implementing strategies that can contribute to improve the quality of life. Specific Objectives were: to determine the maternal mortality of the Siape neighborhood and its causes to implement sociocultural and socio-educational actions that contribute to decrease the situation; Establish the type of controls performed by pregnant women and the treatment indicated in them; To identify the different methods of family planning used by the population of women in the sector for the prevention of unwanted pregnancies. The method used was the quantitative one, with the accomplishment of a research of descriptive type, using a population constituted by the women residing in the Neighborhood Siape of the city of Barranquilla, with ages between the 13 and the 60 years. The sample used was non-probabilistic voluntary. Among the conclusions of the investigation are the following aspects: the most vulnerable communities are reflected the little care of pregnant women, and the faint accompaniment by the relatives in the carrying out of the controls and in the care of them.
\end{abstract}

\title{
Keywords
}

Sexual Health, Reproductive Health, Women, Childbirth, Maternal Mortality.

\section{Introducción.}

La sexualidad en los seres humanos constituye un todo en el transcurso de la vida y en particular en la etapa de la adolescencia. El comportamiento sexual y las pautas reproductivas de adolescentes están influenciadas por su entorno socio cultural, en estrecha relación con su percepción del bienestar psico-afectivo, planteándoles necesidades y cuestionamientos frente a su sexualidad. La manera como dichos cuestionamientos se respondan y como se reemplazan las necesidades, determinan su salud sexual y reproductiva al igual que su vida socioafectiva.

El concepto de salud sexual establecido por la Organización Mundial de la Salud OMS (2004) alude a "la integración de los elementos somáticos, emocionales, intelectuales y sociales del ser sexual, por medios que sean positivamente enriquecedores y que potencien la personalidad, la comunicación y el amor". El 
concepto de salud sexual destaca la significación de aspectos cualitativos para el ser humano, como el desarrollo personal, el vínculo con los otros, el valor de la afectividad y de la comunicación. El concepto de salud reproductiva hace referencia específicamente a los aspectos pro-creativos de la relación de la pareja.

La caracterización de conocimientos y prácticas en sexualidad y reproducción, permiten construir categorías ordenadas sobre los factores que desfavorecen y/o favorecen una buena salud sexual y reproductiva, partiendo del contexto familiar, cultural, educativo y ambiental en el cual se desarrollan.

La sexualidad y la reproducción son parte intrínseca de la salud sexual y reproductiva. La sexualidad forma parte de la vida de las personas durante toda su existencia y se desarrolla a través de los años, sobre la base del conocimiento, valores, creencias y costumbres de su entorno social. La sexualidad es una realidad con la que viven a diario todas las personas, trasciende lo físico (relaciones sexuales); se traduce en las diferentes formas (conductas) que tienen las personas para expresarse y relacionarse con su entorno social y constituye una fuente importante de bienestar y placer tanto físico como mental, pero también constituye uno de los ejes fundamentales sobre los que se construyen las desigualdades e inequidades. Mientras que la sexualidad está presente y forma parte de toda la vida, la reproducción es la capacidad temporal de procrear que tienen hombres y mujeres. En la mujer, esta capacidad está limitada a los días fértiles del ciclo menstrual, en el período comprendido entre la menarquía y la menopausia, mientras que en el hombre esta capacidad es más prolongada.

Este artículo forma parte de los resultados parciales del Proyecto de Investigación que se encuentra en etapa de desarrollo bajo el título Caracterización de la salud sexual y reproductiva de las mujeres del Barrio Siape de la ciudad de Barranquilla, con la dirección de Juan Carlos Berrocal Duran y Rosario Joaquín Reales Vega y la participación de los estudiantes del Programa Trabajo Social, Kevin Patrón 


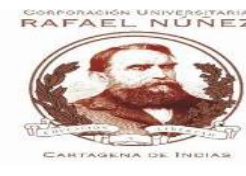

Ayola, Yoismar Cabarcas Vizcaíno y Janeris Mercado Martínez, del Grupo

Vol.8, No 1. Diciembre de 2016 pp. 48 -60

Societas.

\section{Método.}

La investigación es de corte cuantitativa y aborda el uso de fuentes documentales y encuestas no estructuradas, con el propósito de realizar un grado de orientación a conclusiones con un nivel de abstracción de una investigación pura. En cuanto a las dimensiones cronológicas y espaciales, el estudio es de corte sincrónico. El tipo de investigación fue descriptivo y se utilizó como población las mujeres residentes en el barrio Siape de la ciudad de Barranquilla, con edades entre los 13 y los 60 años, por lo que este universo se encontraba en capacidad de aporta información valiosa para el estudio y además por las diversas estrategias implementadas en cuidado de su salud sexual y reproductiva. Se trabajó con una muestra no probabilística sujeto tipo y voluntaria.

\section{Resultados y discusión.}

Las encuestas realizadas al interior de las mujeres seleccionadas, con un tipo de cuestionario con preguntas cerradas con dos opciones, cuyas respuestas fueron sistematizadas como aparecen en las Figuras 1, 2, 3, 4 y 5 las cuales fueron confrontadas con los pronunciamientos que hizo la Corte Constitucional colombiana con respecto a la salud sexual y reproductiva. 
El conocimiento sobre salud sexual y reproductiva de las mujeres del barrio Siape. Los primeros acercamientos

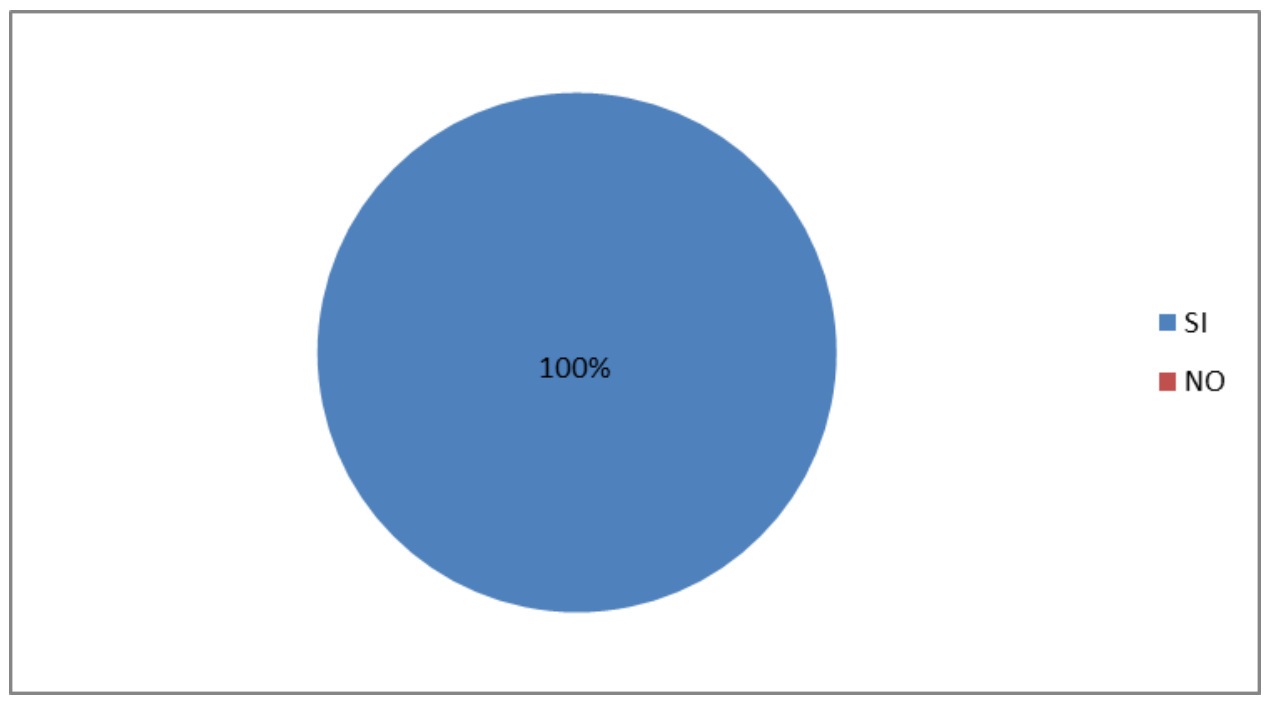

Figura 1. Acompañamiento en el embarazo

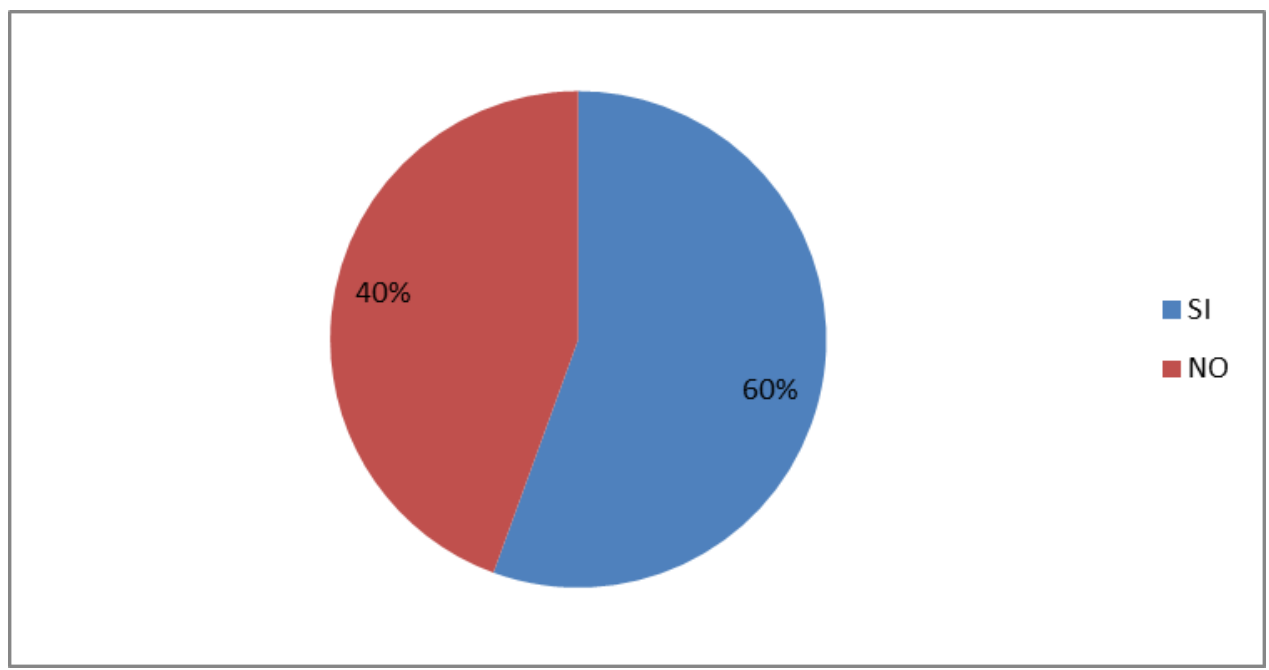

Figura 2. La existencia de otros hijos. 


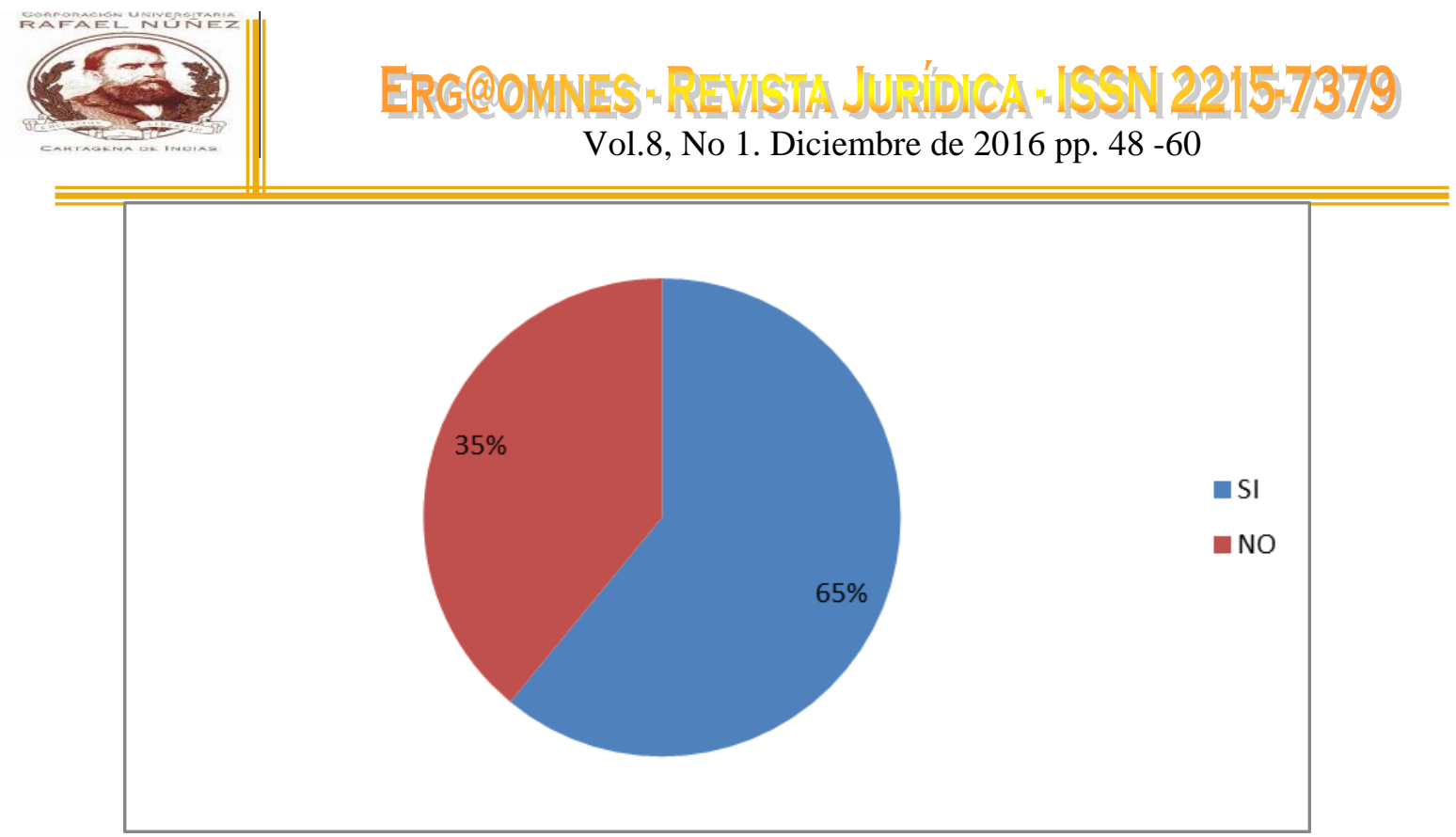

Figura 3. Información sobre la salud sexual y reproductiva.

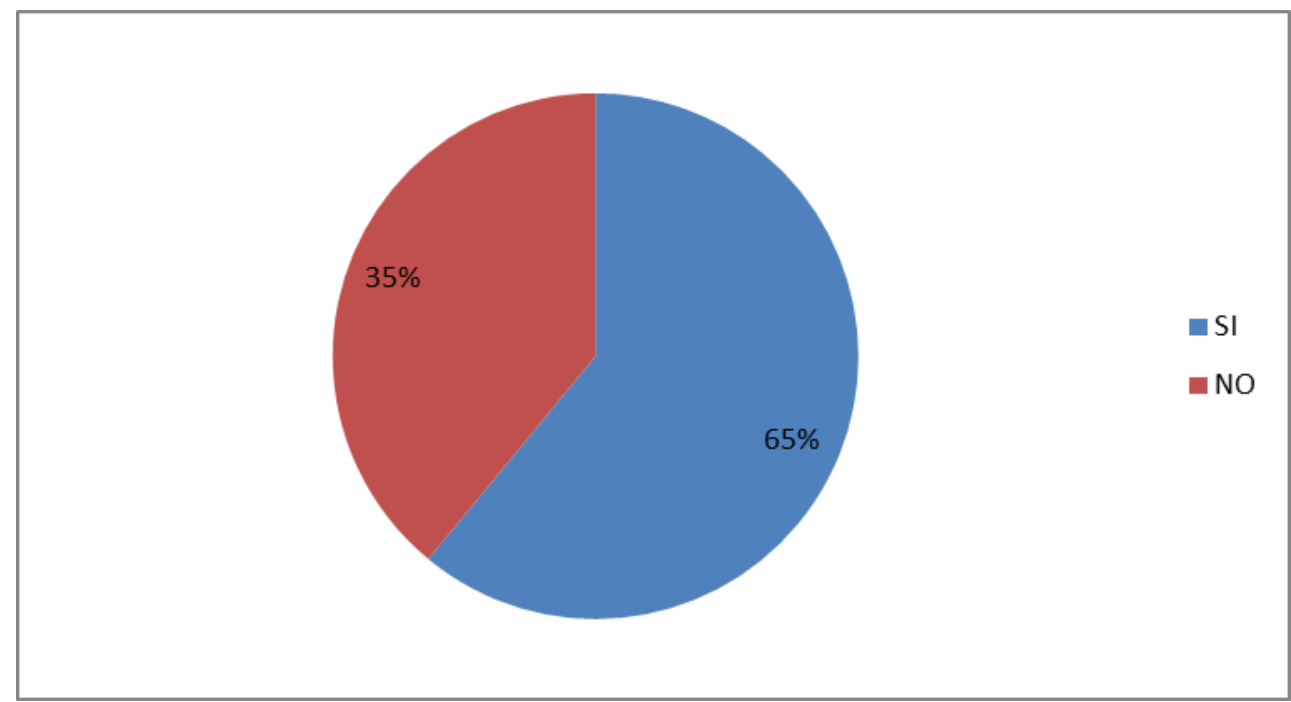

Figura 4. Información sobre Derechos sexuales y reproductivos 


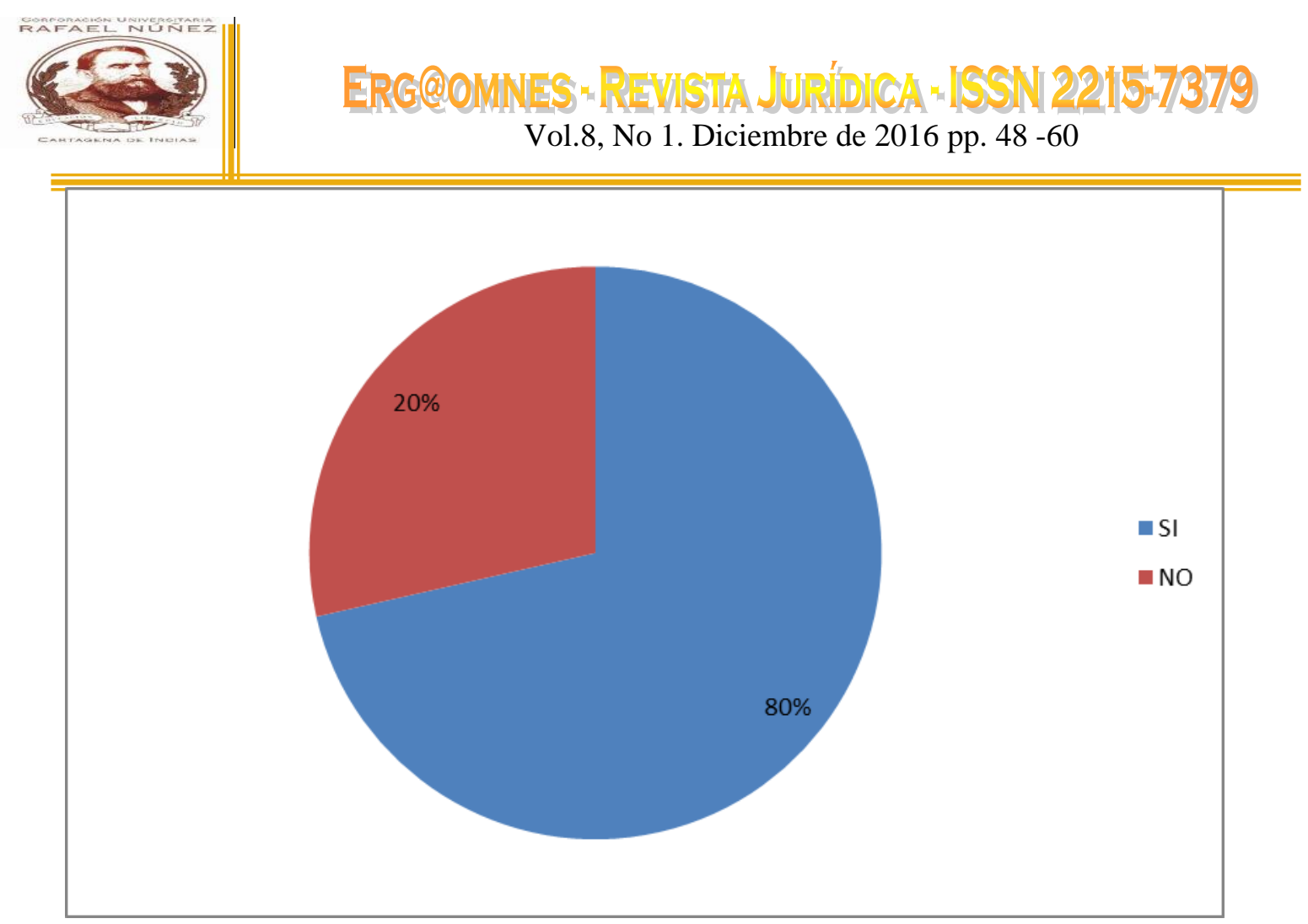

Figura 5. Conocimiento de los padres sobre educación sexual.

\section{Los pronunciamientos de la Corte Constitucional colombiana sobre la salud sexual y reproductiva}

De acuerdo con la literatura relacionada con la sexualidad y la reproducción se saca como conclusión que ambas están íntimamente ligadas a la calidad de vida, desde el punto de vista individual como social. Significa que tanto la salud sexual y reproductiva que en adelante se denominará bajo la sigla SSR, es un "estado general de bienestar físico, mental y social", y no es simplemente la ausencia de enfermedades o dolencias en todos los aspectos relacionados con la sexualidad y la reproducción. También inmiscuye la oportunidad que implica el ejercer los derechos sexuales y reproductivos, que de ahora en adelante se denominan bajo la sigla DSR.

En este sentido, el buen estado de SSR es la capacidad de disfrutar de una vida sexual satisfactoria y sin riesgos, pero también es la posibilidad de ejercer el derecho a procrear o no, la libertad para decidir el número y espaciamiento de los 
hijos, tal como así lo contempló la Constitución colombiana (1991) hace unos 15 años $^{3}$, al igual que lo planteado por el Ministerio de la Protección Social colombiano, al decir:

el derecho a obtener información que posibilite la toma de decisiones libres e informadas y sin sufrir discriminación, coerción ni violencia, el acceso y la posibilidad de elección de métodos de regulación de la fecundidad seguros, eficaces, aceptables y asequibles, la eliminación de la violencia doméstica y sexual que afecta la integridad y la salud, así como el derecho a recibir servicios adecuados de atención a la salud que permitan embarazos y partos sin riesgos, y el acceso a servicios y programas de calidad para la promoción, detección, prevención y atención de todos los eventos relacionados con la sexualidad y la reproducción, independientemente del sexo, edad, etnia, clase, orientación sexual o estado civil de la persona, y teniendo en cuenta sus necesidades vitales.(p. 9)

Ahora, resulta viable en cuanto a contrastar los aspectos que las mujeres del barrio Siape en Barranquilla plantean con las respuestas a las preguntas formuladas en las entrevistas realizadas, por ejemplo, en lo que respecta a los derechos sexuales y reproductivos y la gestión del riesgo que se hace en salud a través de los factores que afectan la salud sexual y la salud reproductiva, que no solamente se conciben como oportunidades a las cuales acceden las personas por el derecho que les compete, sino también por la garantía del acceso de estas "personas a los beneficios de los avances científicos disponibles para el disfrute de la sexualidad y la reproducción y el ejercicio pleno de los derechos sexuales y los derechos reproductivos, como forma de materialización del derecho a la salud física, mental y social", como lo estableciera el Ministerio de Salud y Protección Social (2010), que en otros apartes consideró lo siguiente:

3 La Constitución Política de Colombia (1991) en el artículo 42 hace referencia a que "la pareja tiene derecho a decidir libre y responsablemente el número de sus hijos, y deberá sostenerlos y educarlos mientras sean menores o impedidos", con lo cual se admite que en ese sentido se favorece la salud sexual y reproductiva, con responsabilidad. 
El Estado, especialmente a través de los agentes del SGSSS, debe proveer las condiciones para que todos los habitantes del territorio nacional, hombres, mujeres y personas intersexuales o transgenero, puedan gozar del más alto estándar posible de salud física y mental en el ámbito de la sexualidad y la reproducción, superando restricciones y brechas derivadas de las condiciones de inequidad, reconocidas como Determinantes Sociales de la Salud, para la garantía de los derechos sexuales y los derechos reproductivos, de su ejercicio y del acceso a la atención en materia de salud sexual y salud reproductiva, en la prevención, diagnóstico precoz y tratamiento oportuno de las condiciones que lo requieran. (p. 73-74)

Lo que ha dejado claramente establecido la Corte Constitucional colombiana, mediante Sentencia T-627/12 con relación a la salud sexual y reproductiva es lo siguiente:

Tanto hombres como mujeres son titulares de estos derechos, sin embargo es innegable la particular importancia que tiene para las mujeres la vigencia de los mismos ya que la determinación de procrear o abstenerse de hacerlo incide directamente sobre su proyecto de vida pues es en sus cuerpos en donde tiene lugar la gestación. Los derechos reproductivos están implícitos en los derechos fundamentales a la vida digna.

A la pregunta usted se ha sentido acompañada durante el embarazo, aunque todas respondieron positivamente, queda demostrado que ese acompañamiento probablemente sea de tipo económico, pero no emocional, ya que algunas no conviven con su pareja y el tiempo que le dedican en canto a la prevención y estar pendientes de las situaciones que afectan a su excompañera, no es el adecuado ni tampoco el necesario para el estado emocional que representa un embarazo. De acuerdo con lo establecido por Rovati (2011) esta es una etapa en la cual la mujer sufre una serie de transformaciones ya que durante este "se ponen en marcha complejos procesos hormonales que afectan a casi la totalidad de los 
órganos del cuerpo de la mujer provocando lo que conocemos como síntomas de embarazo" y que de alguna forma inciden en el comportamiento de la persona en esta etapa.

A la pregunta usted tiene más hijos, la mayoría de las encuestadas admitieron tener más hijos y en algunos casos no son de su pareja actual. En estas comunidades en las cuales se evidencian situaciones de marginalidad y desniveles en la educación y la cultura, son propensos a incidir en las decisiones que toman algunas mujeres frente a la decisión de tener hijos con una u otra persona, es decir la no conservación de pareja estable, lo cual repercute en el estado de supuesto abandono o desinterés por parte del hombre.

En cuanto a la recepción de cátedra o charlas relacionadas con la salud sexual y reproductiva, se encontró, de acuerdo con las respuestas suministradas por las mujeres participantes que la mayoría responde en forma positiva y aparentemente están informadas sobre el tema. Las que respondieron negativamente tienen la probabilidad de que en su casa, en la comunidad, ni en ninguna otra parte no se haya escuchado hablar del tema. Es decir, carecen de total información sobre el particular.

Con respecto al conocimiento sobre los derechos sexuales y reproductivos, la mayoría de las encuestadas no supieron explicar la concepción y fueron pobres en el vocabulario dado como ejemplo, del real significado que este contiene. Aunque la mayoría respondió positivamente, al momento de nombrar algunos de estos derechos no supieron explicarlo.

Finalmente, en la batería de preguntas que respaldaban el cuestionario y del ejercicio, cuando se hizo la pregunta, ¿sus padres alguna vez conversaron con usted temas como la educación sexual?, la mayoría hizo alusión positiva y agregaron "que en sus hogares conversaron sobre este tema, lo cual ayudó a resolver dudas, lo que permitió orientarlos", con lo que también despejó las dudas 
del equipo de recolectores de la información, que ya estaban decididos a culminar el trabajo relacionado con la información de fuente primeria.

\section{Conclusiones}

Estos resultados que se pueden tomar en forma parcial, ya que este trabajo continuará en el siguiente semestre, con el fin de determinar la mortalidad materna del barrio Siape y sus causas para implementar acciones socioculturales y socioeducativas que contribuyan a disminuir la situación posibilita la información para seguir indagando sobre el particular y en el desarrollo del mismo, aunque en la Oficina de la Secretaría de Salud del Distrito de Barranquilla, de acuerdo con la información que brinda el Diario El Heraldo (2013):

La Alcaldía Distrital de Barranquilla, por intermedio de la Secretaría de Salud, ha reducido en un $50 \%$ la mortalidad materna, informó esa dependencia, debido a la prevención de enfermedades y atención integral del binomio Madre-Hijo.

A corte del 19 de octubre de 2013, la razón de mortalidad materna ha bajado 17.6, mientras que en el nivel nacional es del 69.3 por cada 100 mil nacidos vivos. A la fecha se han reportado tres fallecimientos de gestantes, mientras que en el año 2012 fueron 6 las muertes en igual periodo. Se han encontrado mayores evidencias con respecto a los objetivos planteados, lo que lo hace pertinente al tratar de establecer si las mujeres embarazadas se realizan controles prenatales y el tratamiento que se les indica en estos es el adecuado.

El Diario El tempo también destacó la información que hace referencia a la disminución de la mortalidad en Barranquilla 
Queda claramente establecido que el acompañamiento que se brinda a las mujeres embarazadas por parte de los familiares solo es económico mas no emocional, ya que algunas no conviven con su pareja y si existe una evidencia de tal convivencia, el tiempo que le dedican a la mujer no es el adecuado ni necesario para el estado emocional que representa un embarazo. Lo que ha ocasionado encontrar hogares donde los hijos no son de la pareja actual.

A pesar de contar con la información necesaria sobre la salud sexual y reproductiva, no logran explicar o definir con claridad que significa salud sexual y reproductiva. A pesar de que aseguran que han recibido suficiente información por parte de sus padres sobre temas como la educación sexual. La mayoría respondió positivamente y afirmaron que en sus hogares conversaron sobre este tema, resolviendo dudas y orientándolos

Lo que da a entender que sobre este tema no existe claridad suficiente y que se debe seguir insistiendo en él y es por esta razón que se decide continuar con este tema de investigación y se entregan resultados parciales, por lo que en algunos despachos judiciales de Barranquilla, las decisiones tomadas por los jueces van en contra vía a lo se plantea constitucionalmente, como bien lo establece Pérez Vásquez (2015) al considerar que si bien existe cierta ambivalencias al momento de hacer el fallo, algunos togados "permiten asegurar que la administración de justicia sea eficaz y que los justiciables recibirán soluciones a sus conflictos, con observancia del debido proceso". (p. 50).

Ya lo había planteado antes Mejía Turizo (1994) en un artículo sobre el particular (Arbitrariedad en las decisiones judiciales y administrativas) al referirse a la actuación judicial o administrativa en cuanto es arbitraria, sobre todo cuando en términos generales los funcionarios que se encuentran en posición dominante hacen uso de la misma para tomar decisiones con las cuales priman los intereses 
por encima de las normas, con lo cual se rompe con los principios de legalidad al debido proceso y equidad, contemplados en la Constitución Nacional que es lo que garantiza la existencia y el respeto hacia los derechos de los ciudadanos, al interior del un Estado Social de Derecho, como del cual presume Colombia.

\section{Referencias.}

Benavides Vanegas, F. (2015). Soberanía, globalización y la constitucion de significado. Justicia, 20(27). https://doi.org/10.17081/just.20.27.811

Bayuelo Schoonewolff, P. (2015). La educación y el Derecho en torno a un nuevo paradigma transformador. Justicia, 20(27). DOI: https://doi.org/10.17081/just.20.27.812

Carrascal, M., Chávez, C., \& Sesin, F. (2014). Correlación diagnóstica de la citología cervical versus colposcopia en lesiones premalignas de cáncer cervicouterino. IPS Universitaria Barranquilla 2013. Biociencias, 9(1), 37-43. Recuperado a partir de http://revistas.unilibre.edu.co/index.php/biociencias/article/view/2838

Clavijo Cáceres, D. (2015). El enfoque de competencias en la formación del abogado para el siglo XXI. Justicia, 20(27). https://doi.org/10.17081/just.20.27.813

Colombia. Asamblea Nacional Constituyente. (1991). Constitución Política 1991. Disponible en: http://www.registraduria.gov.co/IMG/pdf/constitucio-politicacolombia-1991.pdf

Colombia. Ministerio de la Protección Social. Dirección General de Salud Pública. (2003). Política nacional de salud sexual y reproductiva. Bogotá: Ministerio 


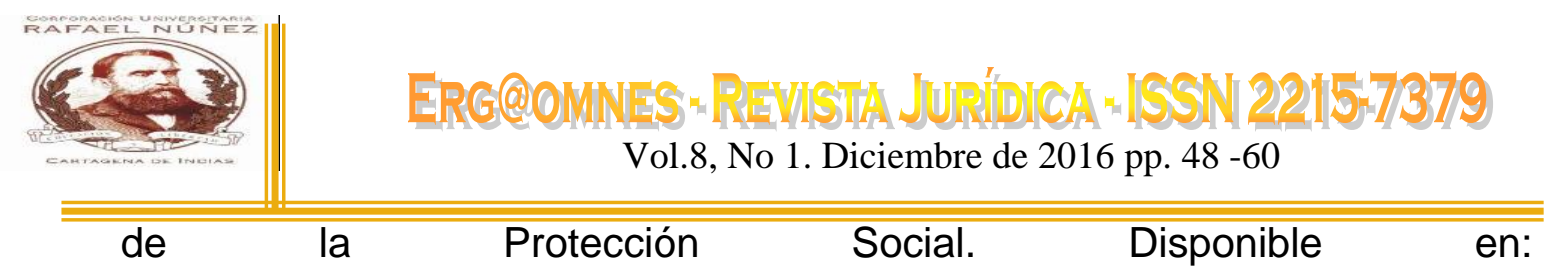

https://www.minsalud.gov.co/Documentos\%20y\%20Publicaciones/POL\%C3 \%8DTICA\%20NACIONAL\%20DE\%20SALUD\%20SEXUAL\%20Y\%20REPR ODUCTIVA.pdf

Colombia. Ministerio de Salud y Protección Social (2010). Política Nacional de Sexualidad, Derechos Sexuales y Derechos Reproductivos. Disponible en: https://www.minsalud.gov.co/sites/rid/Lists/BibliotecaDigital/RIDE/DE/LIBRO \%20POLITICA\%20SEXUAL\%20SEPT\%2010.pdf

El Heraldo. (2013). Barranquilla logra avances significativos en reducción de mortalidad materna. En: El Heraldo (13 de 09 de 2013). Disponible en: http://www.elheraldo.co/local/barranquilla-logra-avances-significativos-enreduccion-de-mortalidad-materna-130006

Escudero Sabogal, I., Jam Arrieta, M., Rodríguez Arrieta, L., Rodríguez Arrieta, L., \& Rodríguez Flórez, M. (2014). Casos de lumbalgia en trabajadores de facturación central- Clínica San Juan de Dios Cartagena-2011. Biociencias, 9(2), 77-85. Recuperado a partir de http://revistas.unilibre.edu.co/index.php/biociencias/article/view/2862

García Cuan, A., Rosado Porto, D., \& Salas Lalinde, M. (2012). Panorama actual de los contaminantes orgánicos persistentes. Biociencias, 7(1), 81-88. Recuperado a partir de http://revistas.unilibre.edu.co/index.php/biociencias/article/view/2796

González Geraldino, F. (2014). Sobre la memoria. Justicia, 19(26). https://doi.org/10.17081/just.19.26.828 
Mejía Turizo, J. (2014). Arbitrariedad en las decisiones judiciales y administrativas. En: erg@omnes. Vol. 6, No. 1 (diciembre de 2014); pp. 150-164. Disponible en:

http://revistas.curnvirtual.edu.co/index.php/ergaomnes/article/view/460/368

Morales, G., Bolaños Contreras, C., \& Larrazábal Ruiz, T. (2011). Enterobacterías aisladas en un centro hospitalario de la ciudad de Valledupar y frecuencia de betalactamasas de espectro extendido y betalactamasas inducibles. Biociencias, 6(2), 33-40. Recuperado a partir de http://revistas.unilibre.edu.co/index.php/biociencias/article/view/2778

Pérez Vásquez, R. (2015). La decisión judicial. En: erg@omnes. Vol. 7, Núm. 1 (2015); pp. 30 -55. Disponible en: http://revistas.curnvirtual.edu.co/index.php/ergaomnes/article/view/460/368

Petro González, I. (2016). La sociologización del pensamiento jurídico. Justicia, 21(29). https://doi.org/10.17081/just.21.29.1232.

Rivera Casas, A., \& Rueda Barrios, A. (2016). Diagnóstico y prevención del parto pretermino. Una actualización de la literatura. Biociencias, 11(2), 55-66. https://doi.org/10.18041/2390-0512/bioc.2.2564

Rovati, Lola. (2011) ¿Qué es el embarazo? Disponible en: http://www.bebesymas.com/embarazo/que-es-el-embarazo

Sánchez Novoa, P. (2015). Hacia un proceso monitorio en Colombia. Justicia, 20(28). https://doi.org/10.17081/just.20.28.1045 